\title{
The Zimm model applied to extended single polymers
}

\author{
Stephen R. Quake \\ Department of Physics, Oxford University, 1 Keble Road, Oxford OX13NP, United Kingdom
}

(Received 9 February 1994; accepted 12 May 1994)

\begin{abstract}
A mean field theory is developed for the Zimm model and compared to the exact solution, computed numerically, for the case of a single extended polymer. An analytic calculation in the dumbbell approximation extends the preaveraging treatment. We find that the dynamic scaling of the preaveraged Zimm model is preserved for short polymers, but that the relaxation function is inhomogeneous in length for long polymers. These calculations can be applied to recent experimental results.
\end{abstract}

\section{INTRODUCTION}

One of the most influential and successful theories of polymer dynamics over the last 40 years has been the Zimm model. ${ }^{1}$ By describing the dynamics of a single polymer, it provides the foundation for theories explaining the macroscopic behavior of polymers. ${ }^{2}$ The various experiments supporting the Zimm model include viscoelastic measurements, ${ }^{3}$ dynamic light scattering, ${ }^{4}$ and measurements of the coilstretch transition. ${ }^{5}$ However, all of these experiments are indirect and depend on a nontrivial amount of further theory and interpretation. Recent developments in the manipulation of single molecules of DNA suggest that DNA is a good model system for directly studying polymer properties, and in fact experimentalists have recently measured the static entropic end-to-end force of a single molecule of $\mathrm{DNA}^{6}{ }^{6}$ as well as the dynamic relaxation of single molecules of stained DNA. ${ }^{7}$ This paper develops a mean field theory of the Zimm model aimed at interpreting the results of single molecule experiments, as well as an extension of the model to nonequilibrium monomer distributions. Computer simulations have been performed to complement the analytic work.

The relaxation experiment was performed by a group at Stanford University and provides an unprecedented direct test of the Zimm model. Optical tweezers were used to manipulate polystyrene beads attached to one end of single DNA molecules. The DNA was stained with a fluorescent dye and visualized in solution with an optical microscope. A fluid flow was used to stretch out the DNA, and then turned off in order to observe the relaxation of the DNA from an elongated state back into a coiled state. The motion of the endpoints was traced with frame-by-frame inspection of the digitized video signal. The authors thus directly observed the dynamical relaxation, instead of looking at correlations of fluctuations about equilibrium. They were also able to put the molecule into the identical initial condition every time they took data. This led to the development of the mean field theory presented in this paper.

The single molecule experiment has several advantages over traditional tests of the Zimm model. None of the other tests excludes interaction with other polymers; indeed, the more dilute the solution the weaker the signal. Even the coil-stretch experiments fail to fully extend the polymer, and recent evidence ${ }^{8}$ suggests that the polymers become only partially aligned. One aspect of the single molecule experi- ment is that since the DNA starts in an extended state, the "preaveraging" approximation in the Zimm model is invalid. This paper treats the hydrodynamics of an extended polymer in order to address the issue of the applicability of the Zimm model.

\section{MEAN FIELD THEORY}

The Zimm model treats a polymer of length $L$ as a series of $N$ beads connected by Hookean springs. The beads each have an effective hydrodynamic radius, and are also hydrodynamically coupled to each other in a solution of viscosity $\eta$. Their motion is modeled by the following set of Langevin equations:

$$
\frac{\partial \mathbf{R}_{n}}{\partial t}=\sum_{m} \mathbf{H}\left(\mathbf{R}_{n}-\mathbf{R}_{m}\right)\left[-k \frac{\partial^{2} \mathbf{R}_{m}}{\partial m^{2}}+\mathbf{f}_{m}(t)\right],
$$

where $\mathbf{R}_{n}(t)$ are the positions of the beads as a function of time, $\mathbf{H}(\mathbf{R})$ is the (nonlinear) Oseen hydrodynamic tensor,

$$
\mathbf{H}(\mathbf{R})=\frac{1}{6 \pi \eta R}(\mathbf{I}+\hat{\mathbf{R}} \hat{\mathbf{R}}),
$$

and $\mathbf{f}_{n}(t)$ is a randomly fluctuating force. In analytic treatments, one typically "preaverages" the hydrodynamic interaction, in which $\mathbf{H}(\mathbf{R})$ is replaced by its average over the equilibrium distribution of polymer states, $\mathbf{H}_{n m} \propto|n-m|^{-1 / 2}$. The equations then become linearly coupled, and can be solved in fourier space, where $\mathbf{H}_{n m}$ is nearly diagonal. One finds that the correlation functions of the eigenmodes decay exponentially, so for example the end-to-end vector

$$
\mathbf{P}(t) \equiv \mathbf{R}_{N}(t)-\mathbf{R}_{0}(t)
$$

has a correlation function

$$
\langle\mathbf{P}(t) \cdot \mathbf{P}(0)\rangle=16 \sum_{p \text { :odd }} \frac{3 k_{B} T}{k_{p}} \exp \left(\frac{-t}{\tau_{p}}\right),
$$

where $k_{p} \propto p^{2}$ and $\tau_{p} \propto p^{-3 / 2}$ are the force constants and relaxation times of the eigenmodes.

This theory predicts correlation functions because the equations are statistical in nature and the experiments addressed have not made direct measurements. The DNA experiment, however, directly measures the end-to-end vector P. Since the initial conditions of the experiment are the same every time, and not randomly distributed, one does not need 
to calculate the correlation function but rather an ensemble average of $\mathbf{P}$. Thus, the experiment measures

$$
\langle\mathbf{P}(t)\rangle=\left\langle\mathbf{R}_{N}(t)\right\rangle-\left\langle\mathbf{R}_{0}(t)\right\rangle,
$$

where the brackets \langle\rangle denote an ensemble average over all relaxations with the same extended initial condition. The term "mean field theory" shall be used to denote any set of equations that describes the evolution of the $\left\langle\mathbf{R}_{n}\right\rangle$ with respect to only averaged quatities. For example, the mean field equations of motion of $\left\langle\mathbf{R}_{n}\right\rangle$ can be found in the preaveraged case by taking the ensemble average of both sides of the Zimm equation,

$$
\frac{\partial\left\langle\mathbf{R}_{n}\right\rangle}{\partial t}=\sum_{m} \mathbf{H}_{n m}\left(-k \frac{\partial^{2}\left\langle\mathbf{R}_{m}\right\rangle}{\partial m^{2}}\right),
$$

where $\mathbf{H}_{n m}$ is the preaveraged Oseen tensor (and hence independent of $\mathbf{R}_{n}$ ) and we have used the fact that the fluctuating force $\mathbf{f}$ has enemble average zero. This is an autonomous set of first order differential equations, whose solution is a superposition of exponential eigenmodes. The solution to the eigenvalue problem of the operator $\mathbf{H}_{n m}\left(\partial^{2} / \partial n^{2}\right)$ is the same spectrum found in the original treatment by Zimm. ${ }^{9}$ However, since the present problem has the well defined initial condition

$$
\mathbf{R}_{n}(t=0)=\frac{n}{N} L \hat{\mathbf{x}},
$$

the coefficients of the exponential modes are determined by this vector, instead of being a thermal distribution of states. In the case $n$ is continuous, this is simply the fourier transform of a function linear in $n$, which behaves like $1 / p^{2}$. Serendipitously, this is the same as the thermal distribution in the $\Theta$-solvent treatment of the Zimm model presented above. When the solvent is "good" and excluded volume effects become important, the power law dependence of the thermal model changes, while the mean field model power law stays the same.

For the preceding example, the mean field theory was exact. To treat the full hydrodynamic tensor, however, requires that one illegitimately bring the ensemble average through nonlinear combinations of the $\mathbf{R}_{n}$. The Zimm equation then becomes

$$
\frac{\partial\left\langle\mathbf{R}_{n}\right\rangle}{\partial t}=\sum_{m} \mathbf{H}\left(\left\langle\mathbf{R}_{n}\right\rangle-\left\langle\mathbf{R}_{m}\right\rangle\right)\left(-k \frac{\partial^{2}\left\langle\mathbf{R}_{m}\right\rangle}{\partial m^{2}}\right) .
$$

In this mean field approximation, fluctuations have been neglected. While this may be partially justified for the case of a $\Theta$-solvent, it almost certainly leads to large errors for good solvents. To see this, image the beads stretched out in a line as their initial condition. Without fluctuation, they will relax along their initial vector, never departing from that line. Excluded volume considerations will thus make them relax to a line segment. In reality, however, fluctuations will cause the beads to depart from the line, allowing them to move around each other and relax to a spherical shape.

For a theta solvent, attempts to integrate the mean field equations led to runaway solutions, due to the unphysical singularity in the hydrodynamic tensor. This was solved by

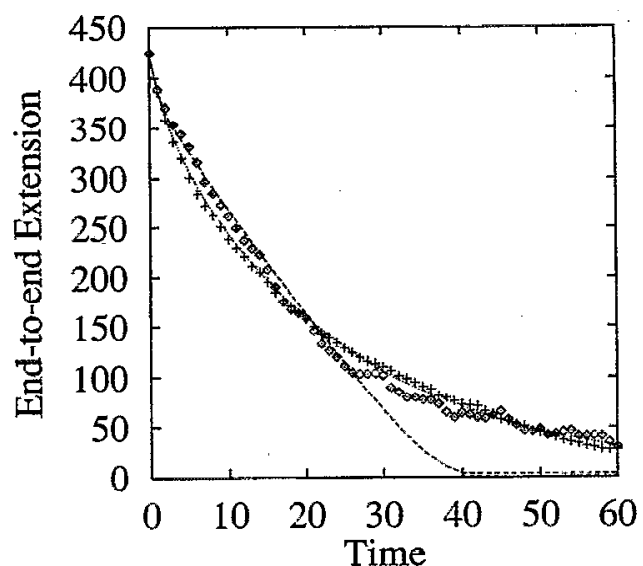

FIG. 1. The relaxation function of a polymer with 50 beads for the preaveraged Zimm model $(+)$ and the full hydrodynamic model $(\diamond)$, compared to the predictions of the corresponding mean field theories (dotted and dashed lines, respectively).

the ad hoc addition of a cutoff to the hydrodynamic tensor when the beads were within one hydrodynamic radius of each other, which allowed the solutions to converge. However, this difficulty is indicative of the breakdown of the point-particle assumptions in the derivation of the hydrodynamics of the Zimm model.

In spite of these caveats, the mean field description is accurate over approximately $80 \%$ of the end-to-end distances during the relaxation, and served to discriminate between the exact and preaveraged Monte Carlo treatments. The equations for 50 beads were numerically integrated and are compared to the Monte Carlo simulations in Fig. 1. The preaveraged system has complete agreement between the mean field theory and Monte Carlo simulation, as expected from the exactness of the mean field theory. The full hydrodynamic system does not agree as well; the relaxation functions agree quite well until the endpoints are about $\frac{1}{5} L$ apart, when they diverge.

\section{THE DUMBBELL MODEL}

The Zimm model can be approximated by neglecting all but the lowest order mode of its relaxation spectrum. ${ }^{10,11}$ This is called the "dumbbell" model, since it is equivalent to assuming the polymer has only two beads connected by one spring. In the mean field approximation, there is only one equation, which is

$$
\zeta(R) \frac{\partial \mathbf{R}}{\partial t}=-k \mathbf{R},
$$

where $\mathbf{R}$ is the end-to-end vector and $\zeta(R)$ is the cumulative mobility of the chain. This mobility has been calculated in a preaveraging approximation, ${ }^{11}$ where it is argued that the functional form is

$$
\zeta(R)=\zeta_{0}\left(\frac{1.2}{1+0.026 E}-\frac{0.17}{E}\right)^{-1} \approx \zeta_{0}(1+0.02 E),
$$




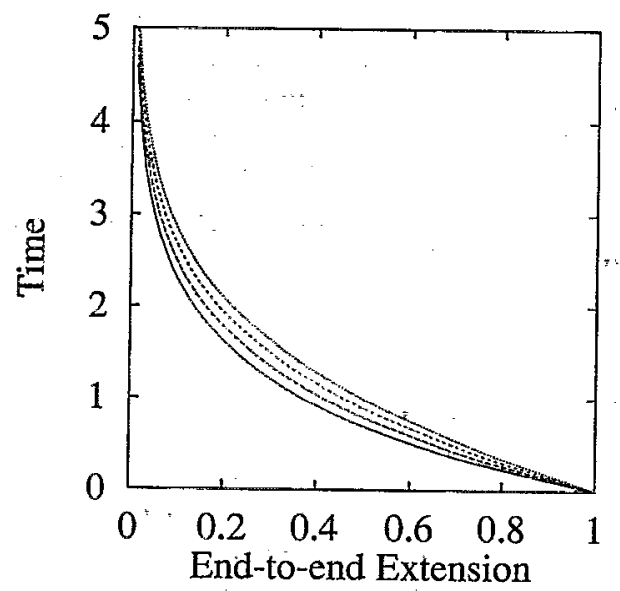

FIG. 2. Comparison of the dumbbell relaxation functions for traditional Zimm model (solid line) and present treatment (dashed and dotted lines). The relaxations have been rescaled in time and extension to demonstrated the inhomogeneity. The traditional Zimm model solutions all collapse onto the solid line, while the solutions from Eq. (12) display increasing inhomogeneity with increasing length. The long dashes correspond to a length of 100 Kuhn lengths, the short dashes to 400 , and the dots to 900. (Note that time is on the vertical axis.)

$\zeta_{0}$ is the constant preaveraged mobility for small excursions from equilibrium, and $E$ is a measure of how stretched the polymer is. In the Appendix we show that if $R_{G}$ denotes the radius of gyration of the polymer, then for $R \gg R_{G}$,

$$
E \approx \frac{R}{R_{G}} \text {. }
$$

The dumbbell equation of motion is then easily solved to yield

$$
\frac{k}{\zeta_{0}} t=-\log \left(\frac{R}{L}\right)+\frac{0.02}{R_{G}}(L-R)
$$

The traditional Zimm model lacks the last term on the righthand side, has relaxation time $\tau=\zeta_{0} / k \propto L^{1.5}$, and is homogeous in $L$. This solution no longer strictly scales with $I_{\text {, a }}$ and the inhomogeneity increases with increasing $L$. For short lengths the function is almost homogenous, and is in fact nearly identical to the dumbbell with constant $\zeta$. Graphs of the solutions for various $L$ are show in Fig. 2.

To estimate how much the scaling law is violated by this family of solutions, the "data collapse" method was used. This method measures scaling relations without reference to a specific model; one relaxation function is rescaled in time and magnitude until it lies on top of another. The rescaling parameters then indicate the relationship of time and length scales. In this case, the initial height of the relaxation function (length of the DNA molecule) and time were rescaled. To make one function "lie on top of" another, an error function (the integrated mean square difference) was minimized. The results are shown in Fig. 3; once the Zimm dependence on $L^{1.5}$ is factored out, the residual nonhomogeneous dependence scales roughly like $L^{0.03}$, a very small change. For longer lengths this error will increase, but for the length range tested in the Stanford experiment the relaxation function obeys the same scaling law as the Zimm model.

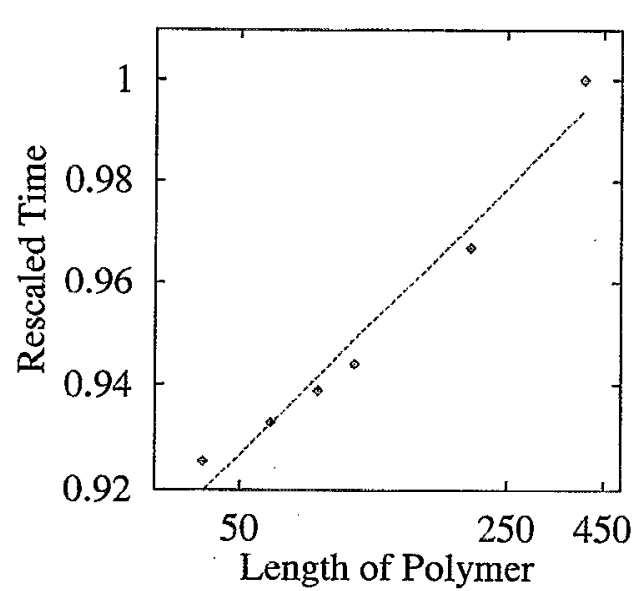

FIG. 3. The inhomogeous residual dependence of relaxation on length for the dumbbell calculation. Once the expected $L^{1.5}$ dependence has been factored out, the inhomogeneity in the data collapse results is small.

\section{COMPUTER SIMULATIONS}

Computer simulations were developed to numerically integrate the solutions to the various models discussed above. Euler's method was used in order to easily incorporate the effects of the randomly fluctuating force. Since the Zimm equations are numerically stiff, care was taken to insure that numerical instabilities did not appear; the simulations were run with a known case of the Rouse model ${ }^{12}$ and compared to the exactly computed solution, which is shown in Fig. 4.

One problem with the Zimm model is that both the exact and the preaveraged Oseen tensor have a mildly singular self-interaction. In the continuum limit, this singularity is integrated away. But in the discrete case, it poses a problem. The usual ad hoc solution is to set the self-interaction equal to the Stokes formula value at the bead's radius. However, this extrapolation is discontinuous, and weakens the long range hydrodynamic interaction. The discontinuity is due to problems with approximating the hydrodynamics of a bead

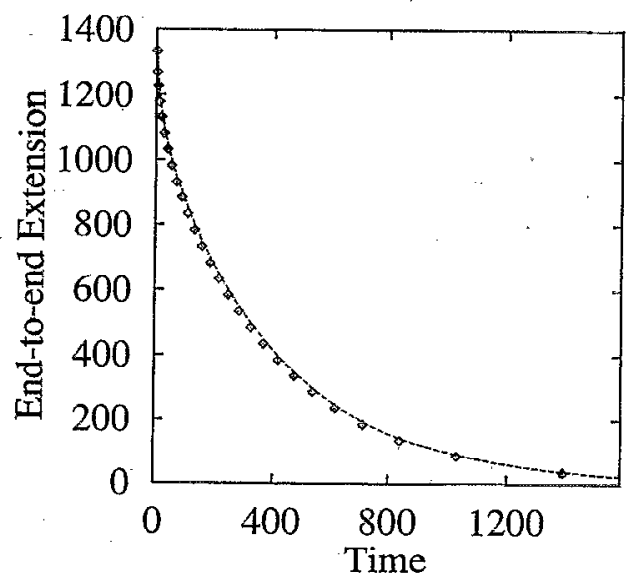

FIG. 4. Comparison of the Monte Carlo of the Rouse model $(\diamond)$ to the predicted analytic function (dashed line) for a polymer with 90 beads. The stiffness of the equations does not cause a numerical instability. 


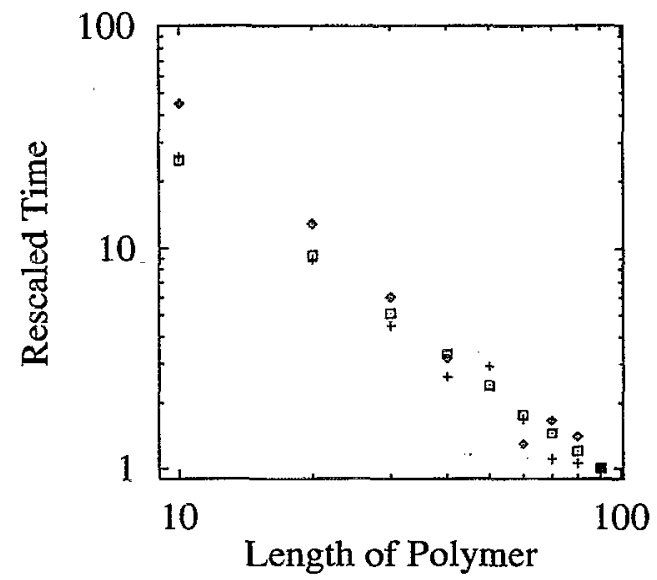

FIG. 5. Comparison of the various scaling laws obtained by data collapse. The preaveraged mean field theory $(\square)$, preaveraged Monte Carlo $(+)$, and full hydrodynamic Monte Carlo $(\diamond)$ all give the expected scaling exponent of 1.5. The length of the polymer is measured in beads $(N)$, where each bead is separated by 15 Kuhn lengths.

extended in space by those of a point particle. We dealt with this problem by normalizing the rest of the tensor to make it continuous with the Stokes self-interaction.

The programs were tested over a range of lengths and temperatures, and the solutions were compared with data collapse to verify that the proper scaling laws were exhibited. Both the Rouse and preaveraged Zimm model yielded the predicted results, as shown in Fig. 5. When the full hydrodynamic tensor was used, the results were virtually indistinguishable from the the preaveraged version. By fitting a loglog plot of the rescaled time vs length to a line, the preveraged mean field simulation gave a power law of $1.473(0.007)$, within $2 \%$ of the predicted value of 1.5 . When the full Langevin equation with fluctuating force was simulated, the power law was $1.48(0.05)$. For the full hydrodynamic interaction, the power law was $1.5(0.1)$, in agreement with the preaveraged results. Although the scaling laws are preserved with the full hydrodynamic interaction, close inspection of Fig. 1 reveals that the functional form of the relaxation is different than that of the preaveraged version. The error bars (not shown) are about the same size as the data points. This difference can also be seen by comparing the corresponding mean field relaxation functions, and coincides with the prediction of the dumbbell calculation that the relaxation function will change but preserve the scaling law on short length scales.

\section{CONCLUSIONS}

The predictions of the traditional preaveraged Zimm model are surprisingly robust, and for short to medium length molecules can be applied to a relaxing polymer far from equilibrium. It has been verified both by analytic calculation and full Monte Carlo simulation that the scaling laws are valid in the regime of the Stanford experiment. For longer lengths, however, the scaling laws break down as the relaxation function becomes inhomogeneous. A mean field theory can describe exactly the preaveraged Zimm model, and is accurate for a large fraction of the initial relaxation of the full hydrodynamic model. This paper has treated the ideal case of a theta solvent, and it will be interesting and nontrivial to extend the results to the excluded volume case in a good solvent.

\section{ACKNOWLEDGMENTS}

The author gratefully acknowledges many helpful discussions with Robin Stinchcombe, and thanks Steven Chu for his hospitality at Stanford, where this work was inspired. He also thanks the British Marshall Commission for financial support.

\section{APPENDIX}

Equation (10) is presented in Ref. 11, where it has been assumed that an extensional fluid flow is deforming the polymer. Although the situation considered here does not have a fluid flow, at the level of the dumbbell approximation one neglects the details of the internal configuration of the polymer, and the important thing is to have calculated a preaveraged mobility for different extensions of the polymer. The definition for $E$ is

$$
E^{2}=\frac{\left\langle R^{2}\right\rangle}{R_{G}^{2}}
$$

For zero flow, $\left\langle R^{2}\right\rangle=R_{G}^{2}$, and $E=1$. It only remains to calculate the relationship between $\left\langle R^{2}\right\rangle$ and $R^{2} \equiv\langle R\rangle^{2}$. We assume a constant force is extending the ends of the polymer, which is represented here by a fluid flow $\mathbf{v}$. The force acting on the ends of the polymer is

$$
\mathbf{F}=-k \mathbf{R}+\alpha \mathbf{v} \text {. }
$$

The energy of the system is $U=-\int \mathbf{F} \cdot d \mathbf{R}$, and the Boltzmann distribution of the end points along $\mathbf{v}$ gives

$$
\left\langle R_{x}^{n}\right\rangle=\frac{\int_{-\infty}^{\infty} R^{n} \exp \left[-\beta\left(\frac{k}{2} R^{2}-\alpha v R\right)\right] d R}{\int_{-\infty}^{\infty} \exp \left[-\beta\left(\frac{k}{2} R^{2}-\alpha v R\right)\right] d R},
$$

where we have assumed $\mathrm{v}=\left(v_{x}, 0,0\right)$. These integrals are easily solved to yield

$$
\left\langle R_{x}^{2}\right\rangle-\left\langle R_{x}\right\rangle^{2}=\frac{1}{\beta k}
$$

The two orthogonal directions $y$ and $z$ follow from similar considerations, and

$$
\left\langle R_{y, z}^{n}\right\rangle=\frac{\int_{-\infty}^{\infty} R^{n} \exp \left(-\beta \frac{k}{2} R^{2}\right) d R}{\int_{-\infty}^{\infty} \exp \left(-\beta \frac{k}{2} R^{2}\right) d R} .
$$


Thus,

$$
\left\langle R_{y, z}^{2}\right\rangle-\left\langle R_{y, z}\right\rangle^{2}=\frac{1}{\beta k} .
$$

The spring constant for a polymer under traction is $k=3 /\left(\beta R_{G}^{2}\right)$, so we have

$$
\left\langle R^{2}\right\rangle=\langle R\rangle^{2}+R_{G}^{2}=R^{2}+R_{G}^{2} .
$$

Then

$$
E=\sqrt{\frac{R^{2}}{R_{G}^{2}}+1} \approx \frac{R}{R_{G}}
$$

when $R \gg R_{G}$.

${ }^{1}$ B. H. Zimm, J. Chem. Phys. 24, 269 (1956).

${ }^{2} \mathrm{M}$. Doi and S. Edwards, The Theory of Polymer Dynamics (Clarendon, Oxford, 1986).

${ }^{3}$ B. M. Crothers and B. H. Zimm, J. Mol. Biol. 12, 525 (1965).

${ }^{4}$ M. Adam and M. Delsanti, J. Phys. (Paris) 37, 1045 (1976); Macromolecules 10, 1229 (1977).

${ }^{5}$ A. Keller and J. Odell, Colloid Polym. Sci. 263, 181 (1985).

${ }^{\circ} \mathrm{S}$. B. Smith, L. Finzi, and C. Bustamonte, Science 258, 1122 (1992).

${ }^{7}$ T. T. Perkins et al., Science 264, 822 (1994).

${ }^{8} \mathrm{G}$. Fuller (private communication).

${ }^{9}$ B. H. Zimm, J. Chem. Phys. 24, 279 (1956).

${ }^{10}$ P. G. deGennes, J. Chem. Phys. 60, 5030 (1974).

${ }^{11}$ R. G. Larson, Rheol. Acta 29, 371 (1990). 